\title{
The utility of DNA extracted from saliva for genome-wide molecular research platforms
}

\author{
Fiona J. Bruinsma ${ }^{1}$, Jihoon E. Joo ${ }^{2,3}$, Ee Ming Wong ${ }^{2,3}$, Graham G. Giles ${ }^{1}$ and Melissa C. Southey ${ }^{2,3^{*}}$
}

\begin{abstract}
Objective: The study aimed to investigate the suitability of DNA extracted from saliva for high throughput molecular genotyping and DNA methylation platforms by comparing its performance with that of DNA extracted from blood. The genome-wide methylation profile, using the Infinium HumanMethylation450 Beadchip array ${ }^{\circledR}$ (Illumina, San Diego, CA), was measured for 20 DNA samples. Common genetic variation was measured, using the Infinium HumanCore Beadchip ${ }^{\circledR}$ (Illumina, San Diego, CA) for 4 samples (matching samples from 2 people).

Results: DNA from blood and saliva returned genotyping call rates and reproducibility frequencies of $>99 \%$. Highquality DNA methylation data was obtained from both saliva and blood DNA, with average detection $\mathrm{p}$-values for each sample ranging from 0.001 to 0.006 . Slightly higher global DNA methylation levels were observed in whole blood DNA than saliva DNA. Correlations between individuals for each sample type were generally greater than correlations between two sample types from the same individual (Pearson's correlation, $r=0.9696$ in 10 pairs of matched blood and saliva derived DNA, $r=0.9702$ between saliva samples, and $r=0.9769$ between blood derived DNA). Saliva yields DNA of sufficient quantity and quality to compare favourably with blood as a source of DNA for genetic and epigenetic research purposes.
\end{abstract}

Keywords: Blood, Saliva, Genetic analyses, DNA methylation, Epigenetics

\section{Introduction}

There is increasing interest in both clinical and epidemiological studies in investigating the genetic and epigenetic markers for diseases and their possible interaction with environmental factors. The collection of blood specimens has enabled studies of circulating cells and other blood fractions (e.g. plasma) and supplied considerable quantities of DNA and RNA for analysis. However, this practice is costly, invasive to the research participant, requires trained phlebotomists and laboratory expertise and infrastructure for sample processing and storage.

Many epidemiological studies have begun collecting saliva samples in addition to, or as an alternative to, the collection of blood, as it can be cost-effective and less invasive. Advantages including; (1) samples collected using commercial kits are stable at room temperature

\footnotetext{
*Correspondence: msouthey@unimelb.edu.au

${ }^{2}$ Genetic Epidemiology Laboratory, Department of Pathology, University of Melbourne, Parkville, VIC, Australia

Full list of author information is available at the end of the article
}

and transportable, (2) self-collection kits can be sent to participant's homes with validated self-guided instructions for providing an adequate sample, and (3) samples can be returned at their convenience. Potential disadvantages of saliva collection include lower mean DNA yield and potential contamination from bacterial DNA $[1,2]$.

Historically, blood samples have been used as a DNA source for high-density molecular platform analysis, although recently DNA extracted from saliva samples have been successfully used for the detection of germline mutations [3, 4] and for measuring single nucleotide polymorphisms (SNPs) [5]. A challenge is that saliva samples contain multiple enzymes and antibacterial components, as well as large quantities of nucleated buccal (epithelial) cells, leukocytes and bacterial DNA [1, 6, 7], potentially making interpretation more difficult. While there has been variation in reported DNA yield from saliva compared with blood $[1,2]$ it has been a sufficient template to enable genetic testing and genotype call rates with high concordance $[1,5,8]$. 
The field of epigenetics has expanded exponentially over the last 15 years. There is increasing interest in the significance of DNA methylation markers to human health. Their potential significance has led to the development of techniques enabling epigenetic markers to be examined across the genome. These methods often rely on the enrichment of methylated DNA using antibodies or methyl-binding substances and most require a large amount of starting DNA [9]. Only one previous study [10] investigated the use of DNA extracted from saliva for methylation analyses.

The Illumina Infinium HumanMethylation450 (HM450K) beadchip array ${ }^{\circledR}$ (San Diego, CA), enables the detection of DNA methylation levels at 485,512 $C p G$ dinucleotides across the genome [11]. It requires relatively small amounts of DNA (as low as $500 \mathrm{ng}$ ) making it appear feasible for use with DNA extracted from saliva [12].

The aim of the study was to investigate the suitability of DNA extracted from saliva and blood for high-throughput molecular genotyping platforms and whether DNA extracted from saliva samples produced data of the same quality as DNA extracted from a blood sample on the HM450K array and the Illumina Infinium HumanCore array ${ }^{\circledR}$. Generation of methylation measurements from DNA extracted from the two sample types allowed us to examine the extent and the nature of the differences in methylation profiles between DNA extracted from blood and saliva.

\section{Main text}

\section{Materials and methods}

\section{Blood and saliva sample collection and DNA isolation}

Blood and saliva samples were obtained from a random sample of 10 participants (approximately $0.5 \%$ of total participants) enrolled in ongoing studies carried out by the Cancer Council Victoria and collected during a 1 month period. Saliva samples were collected using Oragene $^{\circledR}$ (OG-500) saliva collection kits (DNA Genotek, Ontario, Canada). DNA from saliva was isolated using the salt-out method provided by the manufacturer. DNA was subsequently purified using standard ethanol precipitation, eluted in $800 \mu \mathrm{l}-1 \mathrm{ml}$ 1X TE buffer and stored long-term at $4{ }^{\circ} \mathrm{C}$.

Whole blood samples were collected in a $9 \mathrm{ml}$ EDTA Vacutainer (Becton-Dickinson ${ }^{\circledR}$, Franklin Lakes, New Jersey). DNA was extracted from $2 \mathrm{ml}(1 \mathrm{ml} \times 2)$ of whole blood using MagNA Pure automated DNA extraction system (Roche ${ }^{\circledR}$, Basel, Switzerland). All DNA samples were quantified using Quant-iT ${ }^{\mathrm{TM}}$ Picogreen $^{\mathrm{TM}}$ dsDNA assay (Cat No P11496) measured on the Qubit Fluorometer (Life Technologies ${ }^{\circledR}$, Carlsbad, CA) and stored longterm at $4{ }^{\circ} \mathrm{C}$.

\section{Bisulfite conversion and the Infinium HM450K}

Genomic DNA from blood and saliva (500 ng) was bisulfite converted using EZ DNA Methylation-Gold ${ }^{\circledR}$ kit (Cat No D5006) (Zymo Research, Irvine, CA), as per the manufacturer's instruction. $200 \mathrm{ng}$ of bisulfite converted DNA was whole-genome amplified overnight and fragmented. The DNA was precipitated and resuspended in a hybridisation buffer and hybridised onto the HM450K Beadchip overnight. The single-base extension and staining steps were performing using the Freedom $\mathrm{EVO}^{\circledR}$ automated liquid handler (TECAN, Männedorf, Switzerland).

\section{Illumina HumanCore-12 ${ }^{\circledR}$ Beadchip}

Common genetic variation was measured for 4 samples (matching samples from 2 individuals). Genomic DNA from blood and saliva (500 ng)were provided to the Australian Genome Research Facility (Melbourne, Australia) and the Illumina Infinium HumanCore- $12{ }^{\circledR}$ Beadchip assay run as per manufacturer's instructions.

\section{Data processing}

Raw intensity signals from iScan were exported into $R$ environment ( $R$ programming software v3.0.3). The data was processed using the $\operatorname{minf} i \mathrm{R}$ package available from Bioconductor [13]. The data was normalised using the subset-quantile within array normalization (SWAN) to reduce potential technical bias from the platform's two types of probes [9]. Probes with detection p-values $>0.05$ were considered as background noise and subsequently removed. As no sex-specific analysis was anticipated, probes on $\mathrm{X}$ and $\mathrm{Y}$ chromosomes were also removed. $\beta$-values and $\mathrm{M}$-values from a total of 471,899 probes were calculated in the minfi using the formulae: $\beta=$ Meth/ (Meth + Unmeth +100$)$ and $\mathrm{M}=\log ($ Meth/Unmeth $)$.

Raw data from the HumanCore-12 Beadchip was imported into the GenomeStudio v2011.1 Genotyping module 1.9.4 software (Illumina, San Diego, CA) and processed using the software default settings. The Humancore-12v1-0_a manifest and cluster files were used for data quality assessment and analysis as per manufacturer's instructions.

\section{Results}

\section{DNA isolated from blood and saliva}

We successfully isolated genomic DNA from both saliva and blood samples from all 10 study participants. There were 7 males and 3 females aged between 51 and 70 years old at the time of collection. Four had a diagnosis of prostate cancer and six a diagnosis of kidney cancer. A total mean DNA yield of 64.1 $\mu \mathrm{g}$ (range 3.9-176.0 $\mu \mathrm{g}$ ) was obtained from $3.3 \mathrm{ml}$ of saliva, giving a mean yield per $\mathrm{ml}$ of $18.5 \mu \mathrm{g} / \mathrm{ml}$ (range $1.2-44.0 \mu \mathrm{g}$ ). A mean of 
$8.5 \mu \mathrm{g}$ (range 3.2-22.6) of DNA was obtained from $2 \mathrm{ml}$ of whole blood, with a mean yield per $\mathrm{ml}$ of $4.3 \mu \mathrm{g} / \mathrm{ml}$ $(1.6-11.3 \mu \mathrm{g})$.

\section{Measurement of genetic variation}

Based on matching samples from 2 individuals from the Illumina Infinium HumanCore- $12^{\circledR}$ array, both blood and saliva samples returned high quality data with SNP call rates and reproducibility frequencies of $>99 \%$.

\section{DNA methylation data obtained from saliva DNA}

High quality genome-wide DNA methylation data was obtained from matching saliva and blood DNA using the HM450K array. Average detection p-values across all 485,512 probes for each sample ranged from 0.0001 to 0.0006 , and no individual sample had more than 806 probes with detection p-values $>0.01$ (Fig. 1). There was no noticeable difference in data quality between saliva and blood samples. We observed slightly higher global DNA methylation levels in DNA from whole blood samples (average $\beta$-value 0.4963, 95\% CI 0.4899-0.5028) than DNA from saliva (average $\beta$-value $0.4879,95 \% \mathrm{CI}$ $0.4832-0.4928)$, when using average $\beta$-values across all detected probes $(471,899)$ as surrogate measurements.

In order to compare DNA methylation similarities between the two sample types and between individuals, we performed a multidimensional scaling analysis (based on all detected probes). Samples tended to cluster by sample type rather than individuals (Fig. 2). Methylation of DNA from whole blood samples was more uniform between individuals than were the DNA from saliva

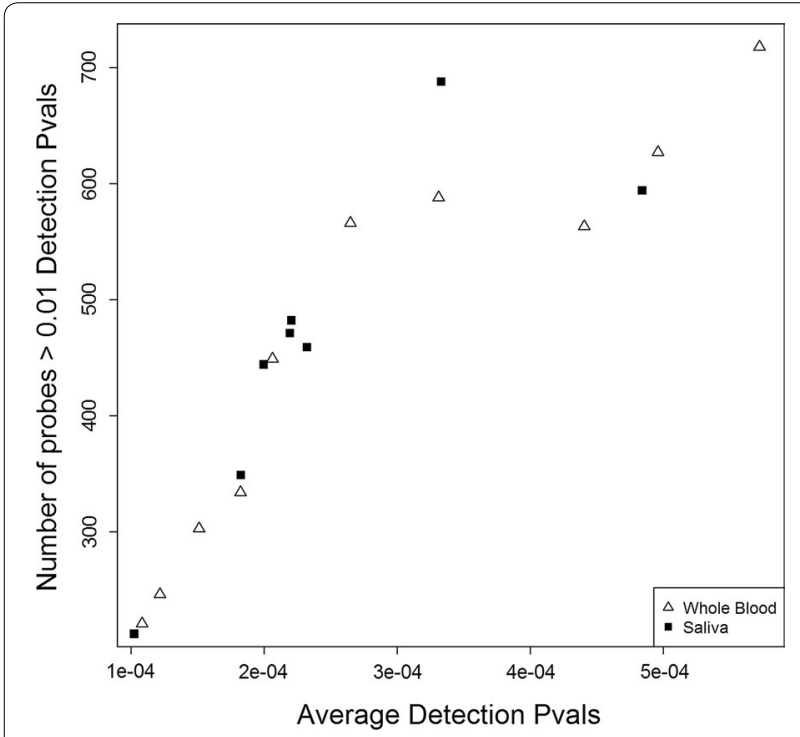

Fig. 1 Plot of average detection $\mathrm{p}$-values and number of probes with $>0.01$ detection $p$-values for individual samples samples. Correlations between DNA of the same sample type were generally greater than correlations between DNA of different sample types (from the same individuals) (Pearson's correlation, $r=0.9696$ in 10 pairs of blood and saliva samples and $r=0.9702$ between all saliva samples, and $r=0.9769$ between all blood samples) (Additional file 1: Table S1).

\section{Tissue-specific DNA methylation marks}

DNA methylation marks of saliva and whole blood samples were found to be highly source specific and we identified a large set of consistently differentially methylated probes between the two source types. An F-test performed on our 10 paired samples found that approximately a quarter of all detected probes $(127,860)$ were significantly differentially methylated (FDR adjusted $p$ value $<0.05$ ) (Additional file 2: Table S2).

\section{Correlative methylation marks between saliva and whole blood derived DNA}

To identify correlative methylation marks between paired DNA sources, we calculated Pearson's paired rank correlation for each of 471,899 probes. There were a large proportion of positively correlated probes between two sources (Fig. 3a). We found 68,870 probes showing moderate to strong correlation between two source types (p-value < 0.01, r > 0.7646) (Additional file 3: Table S3). Only 2712 of these probes were negatively correlated. In order to investigate whether these correlations were biological or a technical artefact of the platform, we checked for overlapping SNPs within these correlative probes. According to the Illumina SNPs annotation table (v3), a large proportion $(25,443)$ of these probes overlapped known SNPs. Given most SNPs are not source-specific, unlike DNA methylation marks, the majority of these correlative methylation marks may have arisen due to the technical limitation (i.e. overlapping SNPs within probes). To investigate this further, the top 9 most correlative probes were plotted (Fig. $3 \mathrm{~b}$ ) and a strong grouping of these samples into 3 groups was observed, suggesting that these methylation signals may actually be driven by underlying genetic polymorphisms. Care should be taken in interpreting DNA methylation results from this platform.

Wu et al. compared a number of methylation markers that are correlative between blood and saliva in young female individuals and found moderate correlation in some markers [4]. We tested four of these markers (cg05575921, cg05951221, cg11924019, cg23576855) on our dataset and we found strong correlations for two probes (cg059512221, $\mathrm{r}=0.9722,95 \%$ CI 0.8830-0.9936; cg23576855, r $=0.9728,95 \%$ CI 0.8858-0.9938; Fig. 3c) (Additional file 4: Table S4). 


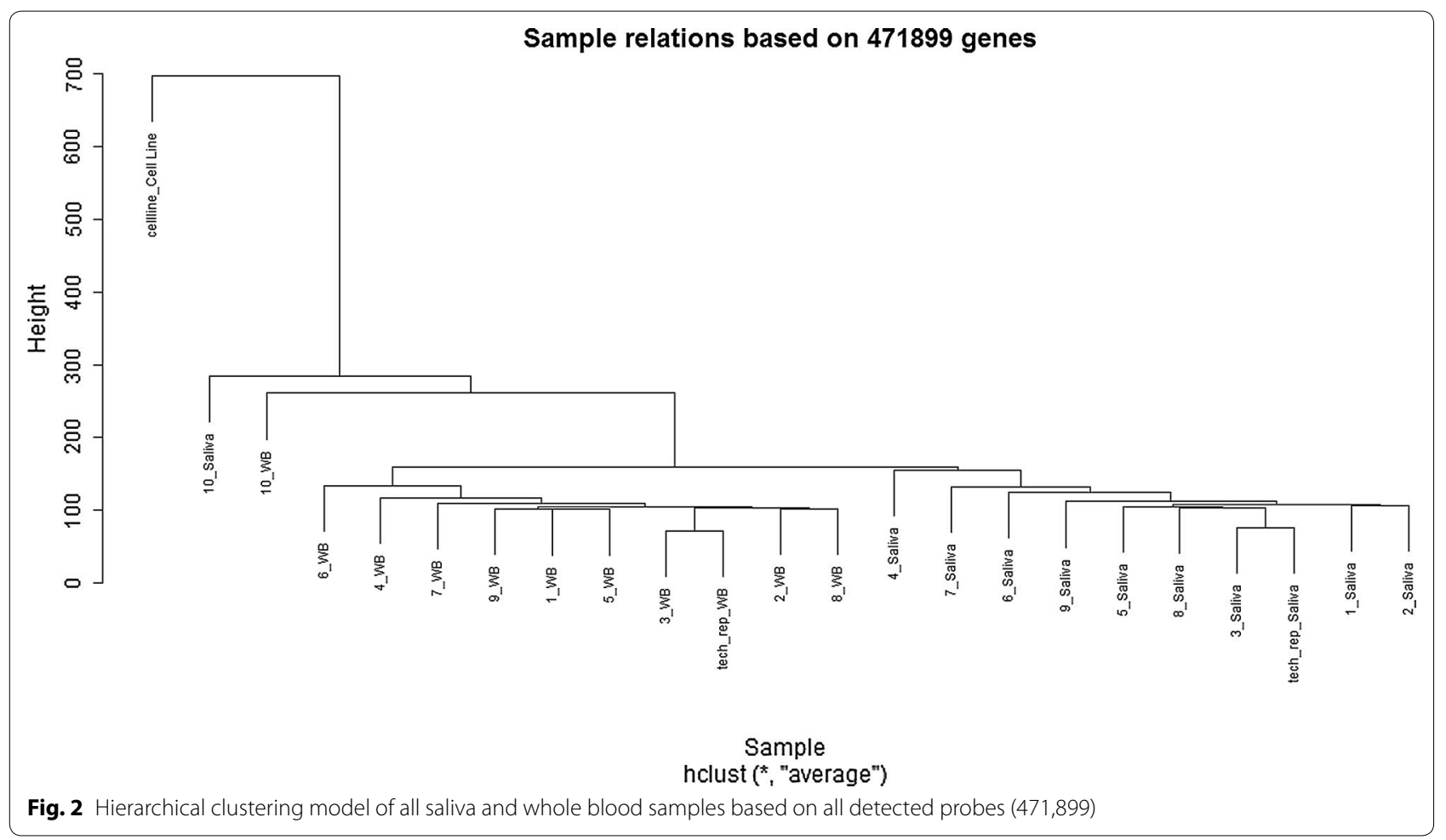

\section{Discussion}

Collecting saliva samples is a non-invasive and convenient method to obtain biological specimens from study participants. The results of our study show that we were able to obtain a higher quantity of DNA from saliva than whole blood samples of the same volume and is consistent with findings reported by Hansen et al. [14]. Current literature $[1,6,8]$ and our broader experience suggests that DNA yields from saliva samples can be quite variable for several reasons including variation in pre-collection mouth content, washing and the DNA extraction method. The use of saliva DNA for a variety of genomic analyses has been previously demonstrated [3-5] and we were able to replicate high call rates on high density SNP arrays consistent with findings from other studies $[1,4$, 15].

The HM450K array data quality matrix of each sample was high and did not differentiate between DNA source. We found that DNA methylation marks were much more similar within each DNA source (Fig. 2). We found almost a quarter of the 471,899 probes were significantly differentially methylated between the two sources, consistent with DNA methylation tissue-specificity [10]. Overall, saliva DNA methylation was slightly more variable than blood derived DNA (Fig. 2 and Additional file 1: Table S1). This is most likely due to the variability in cell composition of saliva samples (they are likely to include a proportion of epithelial and haematological cell lineages). A further study of comparing saliva cell count between samples may be beneficial. However, we believe that DNA obtained from saliva samples is a viable alternative to that derived from blood samples for methylation analyses.

\section{Limitations}

A large set of correlated methylation marks across the source of DNA (within individuals) were identified. Some of these DNA methylation marks may have been identified due to cell type similarities (e.g. leucocytes) or biologically uniform methylation marks between two sources of DNA. The HM450K DNA methylation detection technique is somewhat limited when SNPs overlap 


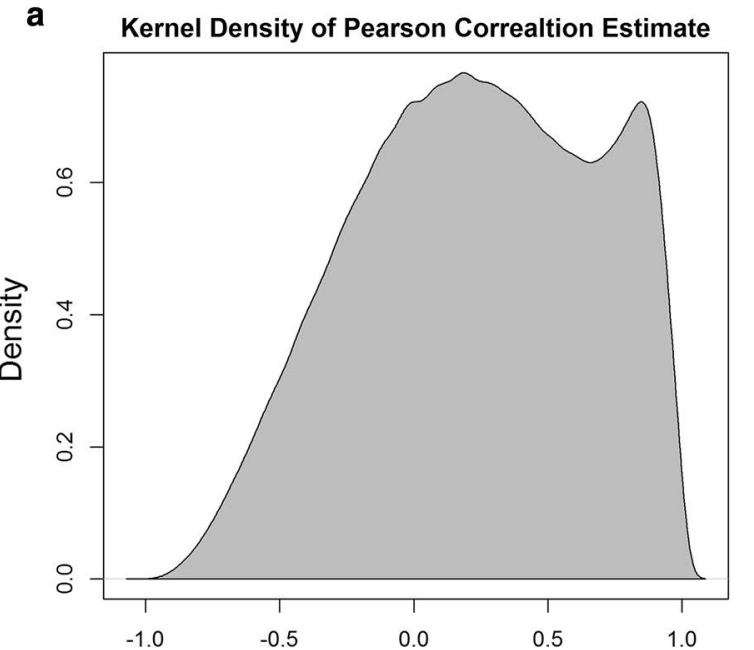

Pearson's Correlation

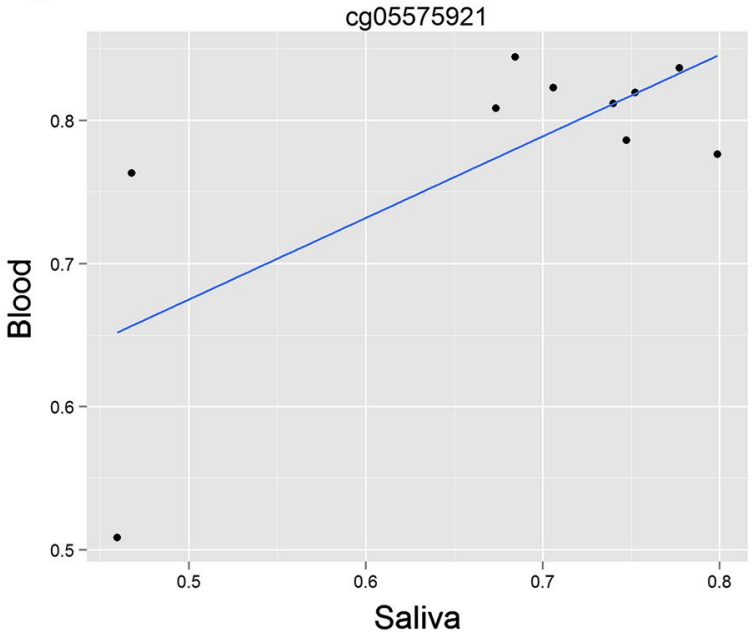

cg05951221

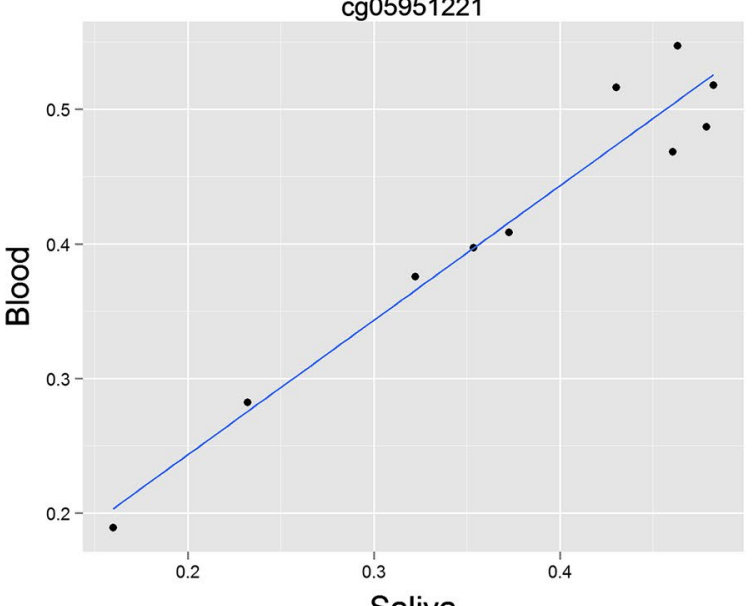

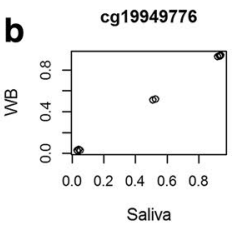
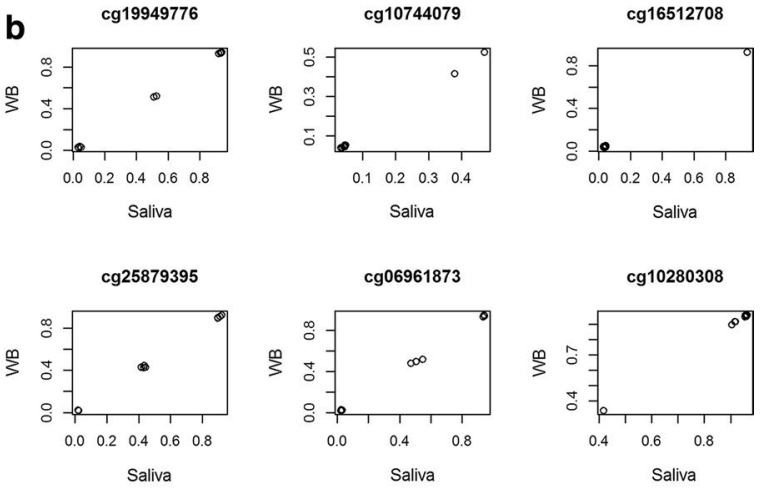

cg18572898
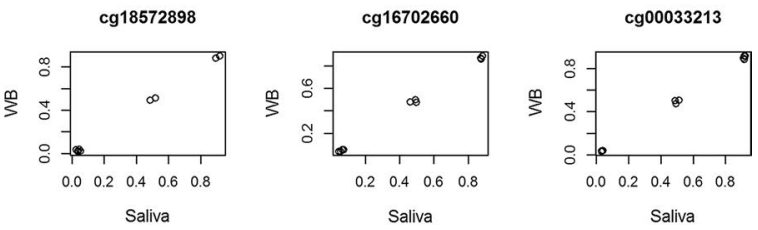

$\operatorname{cg} 11924019$

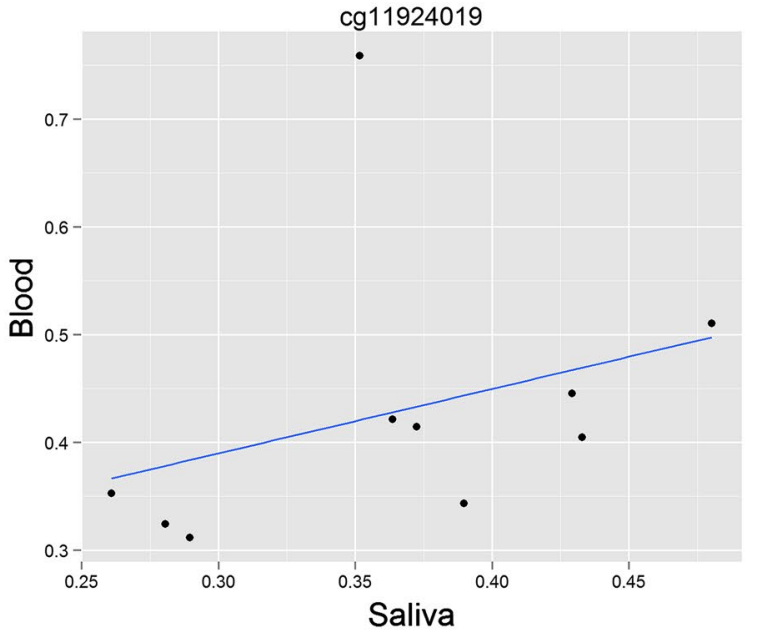

cg23576855

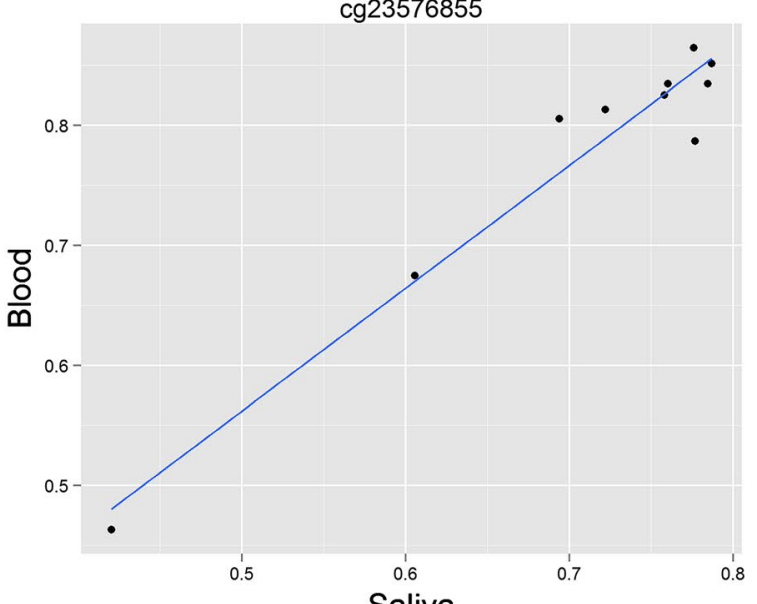

Fig. 3 a Density plot showing pair-wise Pearson's correlation between DNA derived from saliva and blood samples from 10 participants. b Scatter plots of two source types on the top 9 most correlative probes. c Scatter plot showing correlation between two source DNA types on probes cg05575921, cg05951221, cg11924019, cg23576855 
probes which can be misinterpreted as DNA methylation changes. As genetic polymorphisms are uniform across all tissues within individuals a proportion of the correlation between DNAs are due to this technical limitation.

\section{Additional files}

Additional file 1: Table S1. Pearson's correlation coefficient matrix between individual samples.

Additional file 2: Table S2. List of differentially methylated probes (FDR adjusted $p$-value $<0.05$ ) between saliva and whole blood samples.

Additional file 3: Table S3. List of probes showing moderate to strong within-individual correlation between saliva and whole blood samples.

Additional file 4: Table S4. Pearson's correlation coefficient between blood and saliva of probes cg05575921, cg05951221, cg1 1924019, cg23576855.

\section{Abbreviations}

SNP: single nucleotide polymorphism; HM450K: Illumina Infinium HumanMethylation450; SWAN: subset-quantile within array normalization.

\section{Authors' contributions}

FJB, JEJ and EMW were responsible for specimen collection, laboratory bench analysis and data analysis. FJB drafted the manuscript. All authors assisted with data analysis and interpretation and contributed to the final manuscript. GGG is the PI of the studies involved in this report and MCS has overall responsibility for the laboratory work. All authors read and approved the final manuscript.

\section{Author details}

${ }^{1}$ Cancer Epidemiology Centre, Cancer Council Victoria, Melbourne, Australia.

${ }^{2}$ Genetic Epidemiology Laboratory, Department of Pathology, University of Melbourne, Parkville, VIC, Australia. ${ }^{3}$ Precision Medicine, School of Clinical Sciences at Monash Health, Monash University, Clayton, VIC, Australia.

\section{Acknowledgements}

We thank the Australian Genome Research Facility for undertaking the analysis using the Illumina HumanCore Array. We also acknowledge the contribution of study participants for being willing to contribute multiple biological samples for this purpose.

\section{Competing interests}

The authors declare that they have no competing interests.

\section{Availability of data and materials}

The datasets generated and/or analysed during the current study are available from the corresponding author on reasonable request.

\section{Consent for publication}

Not applicable.

\section{Ethics approval and consent to participate}

Ethical approval was obtained from the Human Research Ethics Committee of the Cancer Council Victoria. Research participants provided their informed written consent prior to participation.

\section{Funding}

FJB and GGG are supported by core-funding (raised from charitable donations) from Cancer Council Victoria. Participants and funding for this analysis was provided from National Health and Medical Research (NHMRC) grants 623204 and 1011626. MCS, JJE, MW are supported by the NHMRC grant APP1074383. MCS is a NHMRC Senior Research Fellow (APP1061177).

\section{Publisher's Note}

Springer Nature remains neutral with regard to jurisdictional claims in published maps and institutional affiliations.

Received: 20 August 2017 Accepted: 21 December 2017

Published online: 08 January 2018

\section{References}

1. Abraham J, Maranian M, Spiteri I, Russell R, Ingle S, Luccarini C, et al. Saliva samples are a viable alternative to blood samples as a source of DNA for high throughput genotyping. BMC Med Genomics. 2012;5:19.

2. Gudiseva H, Hansen M, Gutierrez L, Collins D, He J, Verkuil L, et al. Saliva DNA quality and genotyping efficiency in a predominantly elderly population. BMC Med Genom. 2016;2016(9):17.

3. Hu P, Lee CW, Xu JP, Simien C, Fan CL, Tam M, et al. Microsatellite instability in saliva from patients with hereditary non-polyposis colon cancer and siblings carrying germline mismatch repair gene mutations. Ann Clin Lab Sci. 2011;41(4):321-30 (Epub 2011/12/15).

4. Hu Y, Ehli E, Nelson K, Bohlen K, Lynch C, Huizenga P, et al. Genotyping performance between saliva and blood-derived genomic DNAs on the DMET array: a comparison. PLoS ONE. 2012;7(3):e33968.

5. Bahlo M, Stankovich J, Danoy P, Hickey P, Taylor B, Browning S, et al. Salivaderived DNA performs well in large-scale, high-density single-nucleotide polymorphism microarray studies. Cancer Epidemiol Biomarkers Prev. 2010;19(3):794-8.

6. Rogers NL, Cole SA, Lan HC, Crossa A, Demerath EW. New saliva DNA collection method compared to buccal cell collection techniques for epidemiological studies. Am J Hum Biol. 2007;19:319-26.

7. Thiede C, Prange-Krex G, Freiberg-Richter J, Bornhauser M, Ehninger G. Buccal swabs but not mouthwash samples can be used to obtain pretransplant DNA finger printers from recipients of allogeneic bone marrow transplants. Bone Marrow Transplant. 2000;25:575-7.

8. Pulford D, Mosteller M, Briley J, Johansson K, Nelsen A. Saliva sampling in global clinical studies: the impact of low sampling volume on performance of DNA in downstream genotyping experiments. BMC Med Genomics. 2013;10(6):20.

9. Joo J, Wong E, Baglietto L, Jung C-H, Tsimiklis H, Park D, et al. The use of DNA fro archival dried blood spots with the Infinium HumanMethylation450 array. BMC Biotech. 2013;13:23.

10. Wu HC, Wang Q, Chung WK, Andrulis IL, Daly MB, John EM, et al. Correlation of DNA methylation levels in blood and saliva DNA in young girls of the LEGACY Girls study. Epigenet. 2014;9(7):929-33.

11. Maksimovic J, Gordon L, Oshlack A. SWAN: subset quantile within array normalization for Illumina Infinium Human Methylation450 BeadChips. Genom Biol. 2012;13(6):R44.

12. Ilumina Inc. Human methylation 450 bead chip array. San Diego, CA2015. (http://www.illumina.com/products/methylation_450_beadchip_kits. html). Accessed 2 Feb 2015.

13. Aryee MJ, Jaffe AE, Corrada-Bravo H, Ladd-Acosta C, Feinberg AP, Hansen $K D$, et al. Minifi: a flexible and comprehensive bioconductor package for the analysis of Infinium DNA methylation microarrays. Bioinform. 2014;30:1363-9.

14. Hansen TV, Simonsen MK, Nielsen FC, Hundrup YA. Collection of blood, saliva, buccal cell samples in a pilot study on the Danish Nurse Cohort: comparison of the response rate and quality of genomic DNA. Cancer Epidemiol Biomarkers Prev. 2007;16(10):2072-6.

15. Hoffmann T, Kvale M, Hesselson S, Zhan Y, Aquino C, Cao Y, et al. Next generation genome-wide association tool: design and coverage of a high-throughput European-optimised SNP array. Genomics. 2011;98(2):79-89. 